\title{
Contraception for Adolescents
}

\author{
Sarah Mermelstein, $M D^{*}$ \\ Katie Plax, MD
}

\author{
Address \\ "Division of Adolescent and Diagnostic Medicine, Department of Pediatrics, \\ Washington University School of Medicine, 660 S. Euclid Ave, Campus box 8116, \\ Saint Louis, M0, 63110, USA \\ Email: mermelstein_s@kids.wustl.edu
}

Published online: 29 July 2016

(C) Springer International Publishing AG 2016

This article is part of the Topical Collection on Pediatric Gynecology

Keywords Contraception · Teens · Adolescent medicine $\cdot$ Birth control $\cdot$ LARC $\cdot$ Pregnancy prevention

\section{Opinion statement}

Teenage pregnancy rates in the USA have declined for over 20 years, primarily due to increased access to highly effective contraceptive methods. Despite these encouraging trends, US teen pregnancy rates still remain higher than most other developed nations. There are significant medical, psychological, and financial implications and morbidities associated with unintended teen pregnancy and thus primary prevention strategies to decrease rates of teen pregnancy should be a focus for clinicians. New data shows significant benefit of long-acting reversible contraception (LARC)-intrauterine devices or subdermal implants-over shorter acting methods such as the birth control pill. Despite these data, along with policies from the American College of Obstetricians and Gynecologists and the American Academy of Pediatrics supporting provision of LARC to adolescents, there is a lag among clinicians to communicate this information to their adolescent patients as standard of care. Many providers continue to utilize only short-acting methods such as the pill. Until clinicians more comprehensively adopt use of LARC methods as firstline agents for teenagers, rates of unintended pregnancy within this age group will likely remain high. Working to recognize and decrease barriers to contraceptive access should be a public health priority.

\section{Introduction}

\section{Teen pregnancy}

Since its peak in 1991, the US birth rate among teens is down $61 \%$ [1]. Pregnancy rates have similarly declined, which can be attributed primarily to increased use of effective contraception along with lower sexual activity rates within this age group [2]. The teen birth rate in 2014 was 24.2 births per 1000 youth age $15-19$, representing a $9 \%$ decrease from 2013. Despite these improving statistics, US teen pregnancy rate still remains higher than in other developed countries. By comparison, Canadian teenage birth rate is approximately 14 per 1000 or $58 \%$ lower than that in the USA [3]. 
In $2013,67.8$ per 1000 women ages $15-19$ became pregnant or nearly 750,000 young women. At least $80 \%$ of these pregnancies were unplanned [4]. Seventeen percent of births to adolescent mothers were a second or greater pregnancy. Fifty-nine percent of teenage pregnancies end in live births, $14 \%$ end in miscarriages, and $27 \%$ end in abortions. By age 20, 1 in 5 females in the USA will have given birth [5].

Medical, psychological, and economic implications of teenage pregnancy are far reaching. Teen mothers are less likely to receive a high school diploma, more likely to live below the poverty line, more likely to receive public assistance, and more likely to experience mood disorders $[6,7]$. Children born to teen mothers are more likely to be born prematurely, at low birth weight, experience chronic health problems, poverty, neglect, or abuse, and ultimately increased risk of becoming an adolescent parent themselves [8].

\section{Sexual activity among teens}

Along with declining teen pregnancy trends over the last quarter century, sexual activity among teenagers has also decreased. Overall rates of prior sexual activity among youth ages 15-19 have declined by $22 \%$ for males and $14 \%$ for females since the early 1990 s [9].
Despite these downward trends, rates of sexual activity still remain high. According to the 2013 Youth Risk Behavior Surveillance Survey, a poll of high school students ages $15-19,44 \%$ of female and $47 \%$ of male high school students report history of prior sexual activity. Rate of prior sexual activity increases with age, reaching nearly $70 \%$ by age 19 . Mean age of sexual debut for males is 16.8 years and for females 17.2 years. Thirtyfour percent of high school students have had sexual intercourse within the last month [10].

\section{Contraceptive use and trends among teens}

Most teens report use of contraceptives with sexual activity at least once, most commonly condoms ( $97 \%)$ or the birth control pill (54\%) [5]. Nevertheless, teens are less likely to use a method consistently than adults. When asked if a contraceptive method was used with last sexual activity, $13.7 \%$ of teens reported no method was used, $59.1 \%$ reported condom use only, and only $25.3 \%$ used some form of hormonal contraceptive (OCP, IUD, implant, shot, patch, or ring). See Fig. 1. A mere $8.8 \%$ of teens reported dual use of both a condom and hormonal contraceptive with last sexual activity. These data are a striking reminder that many teens remain at risk for unintended pregnancy.

\section{Clinical approach}

\section{Consent, confidentiality, and counseling}

Clinicians who serve adolescents play a critical role in helping youth recognize and understand safer sex practices and in providing access to comprehensive reproductive health care. Creating an environment where youth feel safe will increase opportunity to provide important preventive counseling and interventions, including contraception.

It is important to inform patients of your consent and confidentiality policies. Many adolescents may not be aware that they are able to consent for confidential health care related to sexual health and contraception without parental involvement. A clinician needs to be familiar with local laws regarding minor consent related to contraception, as these laws are variable from state-tostate [11]. For states without specific policy, federal statutes and best-practice guidelines support minor consent and confidentiality surrounding contraceptive management. Title $\mathrm{X}$ funded family planning clinics are required to provide confidential services to adolescents [12].

One of the primary barriers to youth accessing contraception is concern about confidentiality. A study among minors in a family planning clinic revealed that $59 \%$ would stop going to the clinic if parental notification was required [13]. While the Health Insurance Portability and Accountability Act (HIPAA, [Pub L No. 104-191,1996]) allows parents/guardians access to a minor's records, it also specifically denies release of a minor's information when it 


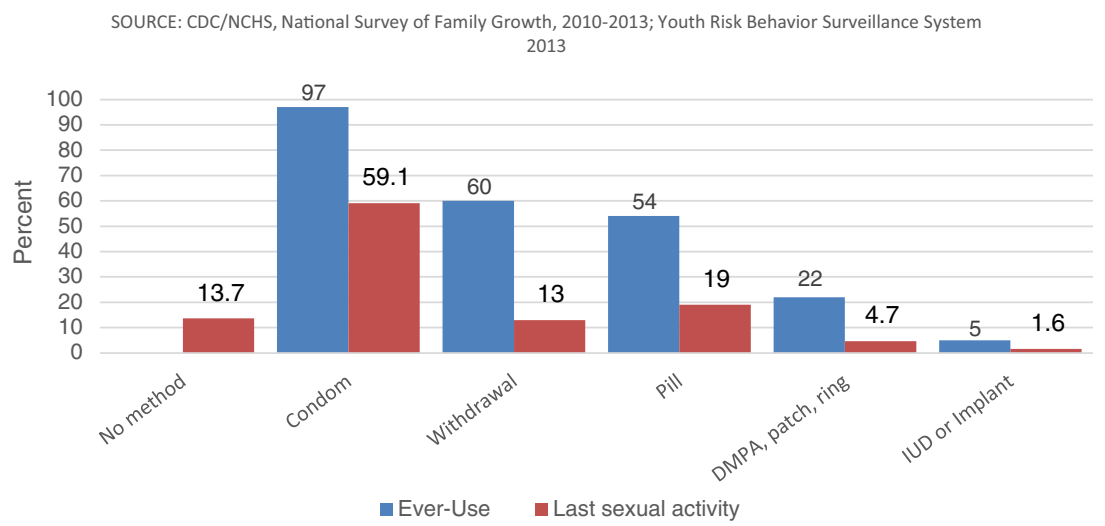

Fig. 1. Contraception usage among teens. Ever-use vs. Last sexual activity.

pertains to medical care that the minor themselves can consent to, such as sexually transmitted disease testing/treatment and often contraceptive counseling and management [14]. This allows clinicians to assure their patients that these services cannot be discussed or released to parents without specific permission from the minor themselves.

Providing adequate education and follow-up to ensure appropriate use and adherence is critical in achieving optimal levels of efficacy. It is also important to dispel myths or misunderstandings about contraception, as patients may make decisions regarding contraceptive use based on false information. Common issues include concerns about long-term fertility impairment, cancer risks, weight gain, or interference with natural cycles, belief that the pill should be first line for everyone, or that long-acting methods are reserved for married or monogamous older women. Unless the misinformation or preconceptions are elicited by the clinician, it may hinder contraceptive decision-making.

When providing contraceptive counseling, clinicians should remind adolescents that abstinence is $100 \%$ safe and effective at preventing unintended pregnancy. In addition, given high rates of sexually transmitted diseases in adolescents, safer sex practices with barrier protection (condoms) should be encouraged with all intercourse [15]. Finally, when counseling patients regarding risks and potential side effects that may occur with contraceptive use, patients should be reminded that every method of contraception is safer than pregnancy.

\section{Identifying and decreasing barriers}

Barriers to contraceptive access are numerous, including cost, concerns about confidentiality, fear of side effects, partner disapproval, lack of transportation, or substance use with sexual activity. Many teens do not believe they can get pregnant or they may desire pregnancy. Clinical visits offer key opportunities for clinicians to discuss these concerns and barriers with their patients in order to try to overcome them.

Under the federal Patient Protection and Affordable Care Act, birth control has been considered a recommended preventive service since 2011. This means that under the ACA, all FDA-approved birth control methods are covered by insurance companies without co-pays or deductibles. This includes private and government-funded insurance programs. At time of publication, there remain exemptions for some insurance plans to cover birth control under ACA, 
including plans in existence prior to 2012 and religiously affiliated institutions. The benefits of ACA coverage also exclude youth without insurance.

\section{Contraceptive methods}

\section{Overview}

The goal of contraception in adolescents is a safe and effective method of preventing pregnancy that is both convenient and reversible. When choosing a method, it is important to recognize the distinct advantages and disadvantages of each method and to consider the following factors: efficacy, ease of dosing/ duration of action, impact on menstrual bleeding, time to return of fertility, side effects, cost, non-contraceptive benefits, and medical contraindications. A comprehensive medical history should be taken-including past medical history, family history, and current medications-to assess for possible contraindications that would limit eligibility for certain types of contraception. The World Health Organization and Centers for Disease Control and Prevention each have resources for eligibility criteria for contraceptive use [16, 17]. The CDC also has a mobile tool for easy access to their medical eligibility criteria for contraception (CDC contraception app).

Contraceptive methods that are less user-dependent have significantly higher efficacy, particularly in the adolescent population. The contraceptive failure rates of birth control pill, patch, and ring have been found to be more than 20 times higher in the adolescent population than LARC methods [18]. As a result, the American College of Obstetricians and Gynecologists (ACOG) and the American Academy of Pediatrics (AAP) both support use of LARC methods as first-line contraceptives for adolescents $[19,20]$. ACOG considers LARC methods "top-tier contraceptives based on effectiveness...the best reversible methods for preventing unintentional pregnancy, rapid repeat pregnancy and abortion in young women." The AAP encourages pediatricians to "discuss the most effective contraceptive methods first." Studies have shown that if barriers such as cost and access are removed, teens and young women prefer LARC methods nearly $70 \%$ of the time over shorter-acting, less effective forms of contraception. Additionally, teen LARC users were more likely to continue their method and had higher satisfaction rates over non-LARC methods [18, 21].

See Table 1 for summary of contraceptive methods.

Historically, providers have counseled patients to wait until the first day or first Sunday of next menstrual cycle to initiate a new contraceptive method. This strategy is no longer considered best practice as unintended pregnancy may occur in the interim. Instead, a "quick-start" method is typically indicated [25]. This allows initiation of contraception as soon as pregnancy has been reasonably ruled out. This process requires clinicians obtain a urine pregnancy test, date of LMP, and date of last unprotected sexual activity and offer emergency contraception if indicated. If unprotected sex has occurred since LMP, urine HCG should be considered inconclusive. In this case, patients may still elect to initiate a hormonal contraceptive right away as there is no evidence of teratogenicity of these medications. A pregnancy test 


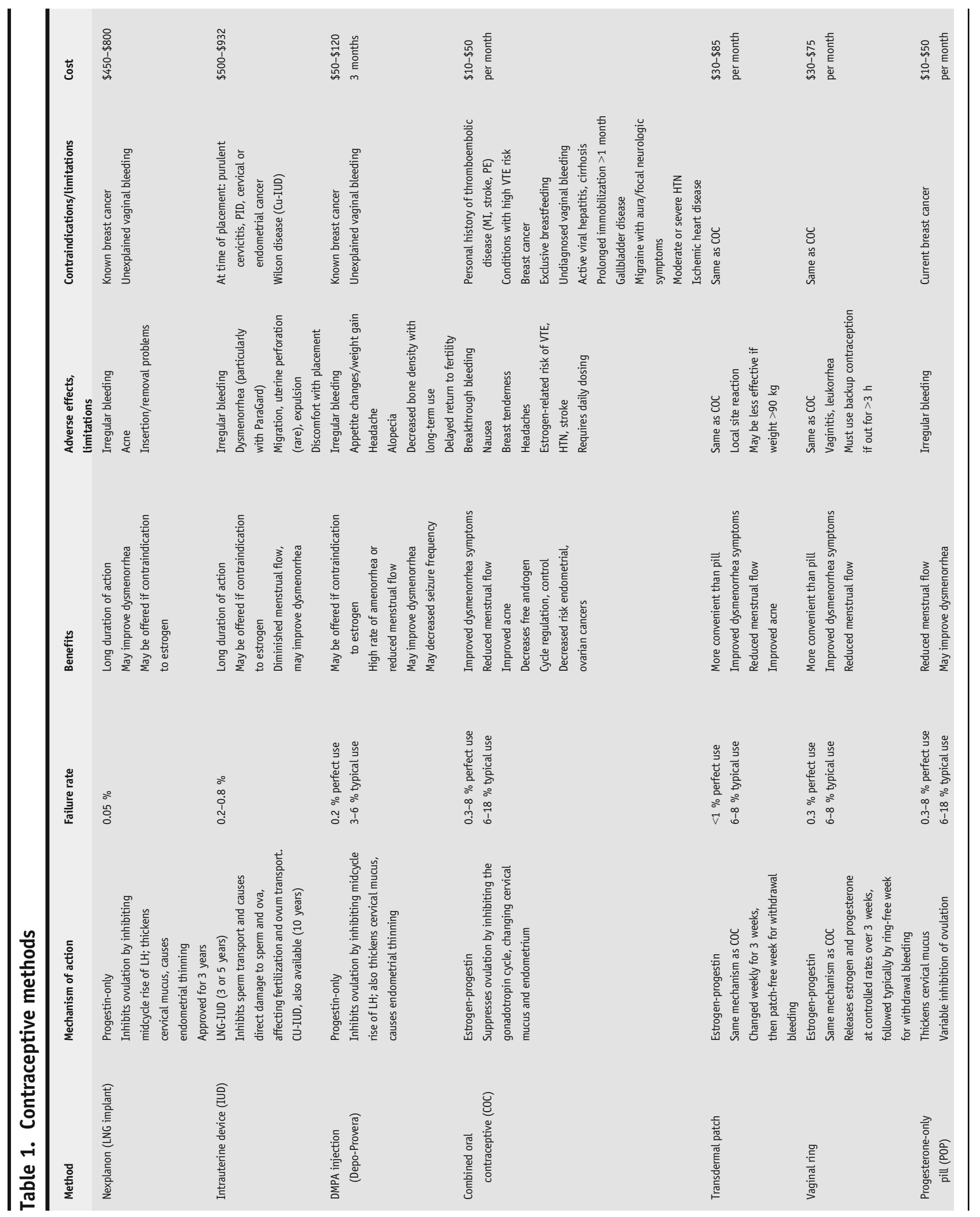




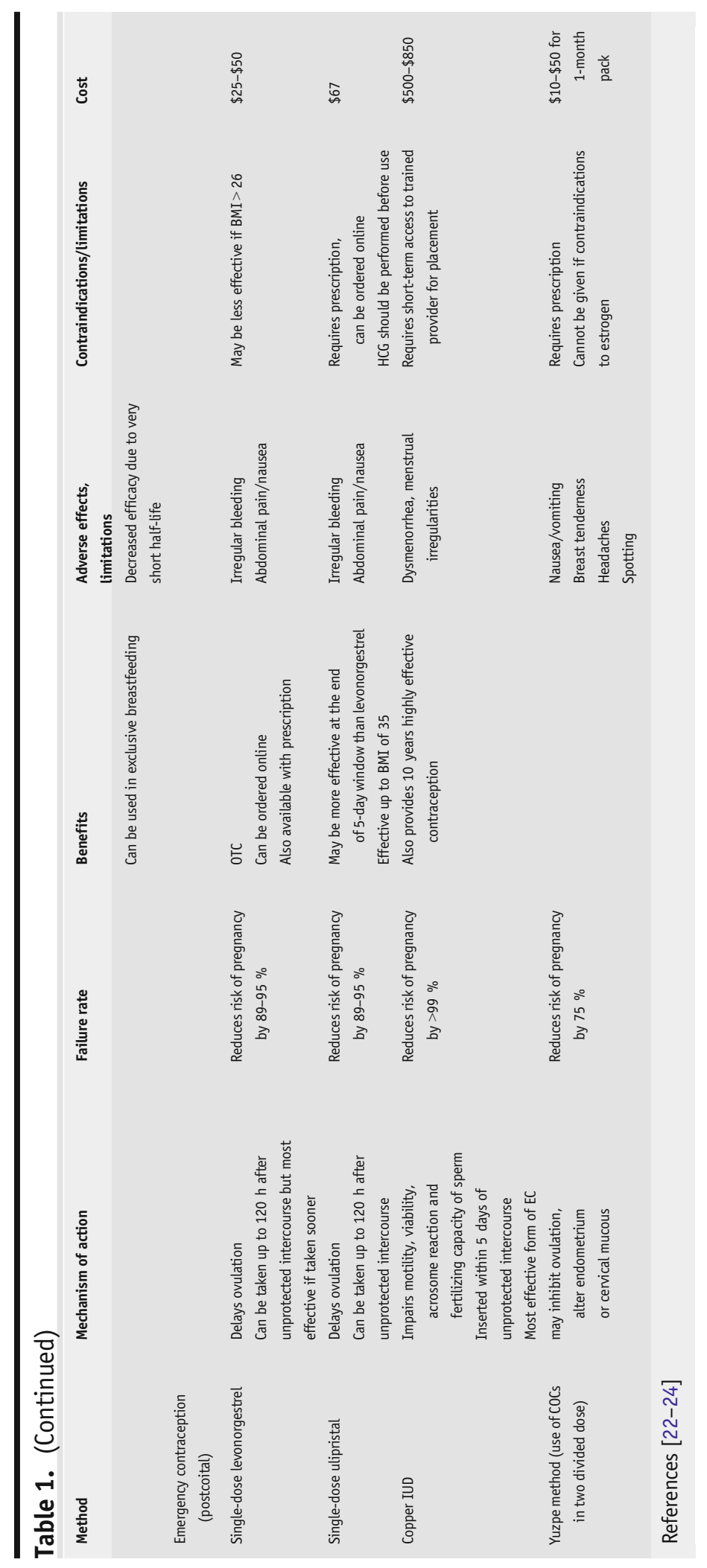


should be repeated 2 weeks later. After quick-start initiation, a backup method should be used for 7 days after initiation. Quick-start method may be utilized for LARC methods if patient has a negative pregnancy test and no unprotected sex since LMP. If unprotected sex has occurred since LMP, a bridge method (OCP, patch, ring, DMPA) should be offered.

In asymptomatic women, a pelvic exam is not indicated prior to prescription and initiation of a contraceptive method, with exception of IUD placement.

\section{Progestin implants}

The contraceptive implant (Nexplanon; Merck), is the most effective form of contraception on the market. It is a $4 \mathrm{~cm} \times 2 \mathrm{~mm}$ implantable rod that contains a progestin, etonogestrel. The device is currently FDA approved for 3 years. The primary mechanism of action is inhibition of ovulation.

Subdermal placement in the nondominant arm is performed by a trained provider and requires local anesthetic. Removal can be performed at any time, up to 3 years after insertion.

The most common side effect with progestin implants is irregular, unpredictable bleeding. While many women see either reduction or cessation of menstrual bleeding (33\%, $22 \%$, respectively), as many as $23 \%$ of users may experience frequent or prolonged bleeding [26]. This side effect is the primary indication for premature removal. Troubleshooting for irregular bleeding with the implant can include treatment with NSAIDs, ethinyl estradiol (if endometrium is suspected to be atrophic), or oral progestin (if endometrium is thickened).

There are currently four FDA-approved intrauterine devices. Three contain levonorgestrel (LNG) (Mirena, Bayer, approved for 5 years; Liletta, Allergan, approved for 3 years; Skyla, Bayer, approved for 3 years). Skyla is the smallest IUD ( $28 \mathrm{vs} .32 \mathrm{~mm}$ ) and is the first to be FDA approved in nulliparous women. Liletta is the newest on the market and offers the advantage of lower cost. The 10-year copper IUD (Paragard, Teva) is a hormone-free option for women with contraindications or who prefer to avoid hormones.

After insertion of a LNG-IUD, unpredictable bleeding may occur for the first 3-6 months; however, most women will see overall reduced menstrual bleeding thereafter. After 12 months of use, approximately one third of women will experience amenorrhea with the Mirena or Liletta [27]; $6 \%$ for Skyla [28]. The Cu-IUD, alternatively, is associated with increased duration and volume of menstrual bleeding.

IUD placement requires a trained provider. STI testing should be performed at the time of placement, but placement can proceed as long as there is no clinical evidence of infection. Contrary to past beliefs, rates of pelvic inflammatory disease (PID) in adolescent IUD users over the life of the device are comparable to non-IUD users [29].

\section{Depo-medroxyprogesterone acetate (DMPA)}

DMPA (Depo-Provera; Pfizer) is an injectable progestin administered at 11-13week intervals. It may be given in a single dose of $150 \mathrm{mg}$ IM or $104 \mathrm{mg}$ 
subcutaneously. With perfect use, efficacy is similar to LARC methods; however, higher typical use failure rates likely reflect patients who do not receive subsequent doses within the therapeutic window.

Nearly all women experience changes to the menstrual cycle with DMPA use. Frequent or prolonged bleeding may occur, particularly in the first 6 months of use. Intervention strategies include NSAIDs or ethinyl estradiol. With ongoing use, most women see decreased menstrual bleeding over time. Fifty percent of women are amenorrheic after 1 year of use and $80 \%$ after 5 years [30].

In some patients, DMPA causes increased appetite and subsequently weight gain. Diet and exercise regimen should be a point of counseling at all visits for patients who are overweight or obese.

Another notable side effect is potential for decreased bone mineral density, particularly after prolonged use. For those without other risk factors, it is generally accepted that the contraceptive benefits outweigh the risks of bone changes, as these changes are reversible upon discontinuation and have not been associated with increased fracture or growth impairment [31]. Clinicians may encourage patients to take calcium with vitamin $\mathrm{D}$ and engage in weight-bearing activity while receiving DMPA, as these efforts promote general bone health, although no specific studies have shown any measures that specifically offset bone loss in DMPA-users.

DMPA has the longest return to fertility after discontinuation among hormonal contraceptives. While most women will return to baseline ovulatory function after 4-6 months, it can take up to 9-18 months for some.

\section{Combined estrogen-progestin methods}

\section{Contraindications}

There are a number of additional contraindications for combined estrogenprogestin methods as compared to progestin-only contraceptives. Most commonly, conditions with high venous thromboembolism risk (SLE, antiphospholipid antibody syndrome, protein C/S deficiency, antithrombin 3 deficiency, factor $V$ Leiden thrombophilia), migraine with aura/focal neurologic symptoms, moderate or severe HTN, history of ischemic heart disease, or medication interaction. See Table 1 and WHO/CDC eligibility criteria for more details.

VTE risk

Exogenous estradiol, like endogenous estrogen, increases risk for venous thromboembolism (VTE). The risk with COC/patch/ring use, however, remains lower than with pregnancy. General adolescent population statistics reveal VTE risk of approximately 1/10,000 woman-years per year; with COC use 3-4/10,000 woman-years; in pregnancy or postpartum 10-20/ 10,000 woman-years [20]. Patients should be screened for additional risk factors (personal or familial thrombophilia, migraine with aura or focal neurologic symptoms, cigarette use, prolonged immobilization) prior to prescription of a combined contraceptive method. 
Combined oral contraceptives (COC) remain the most frequently prescribed form of contraception, despite the fact that they are associated with the lowest efficacy rates. Failure rates among adolescent COC users $(<21$ years of age) have been shown to be twice as high as in older users. Fixed-dose or phasic regimen COCs are currently available. Monophasic pills (stable dose of estradiol and progestin components in all active pills) are most frequently used. The estradiol component is used to establish dosing, typically 20,30 , or $35 \mathrm{mcg}$. Traditionally, women take 21 to 24 days of "active" pills (hormone-containing) followed by 3-7 days of placebo (hormone-free) pill to induce withdrawal bleeding. Some women may prefer to utilize an extended cycle ("stacking") in which placebo pills are skipped and continuous exposure to active pills occurs, thereby avoiding a withdrawal bleed. Most women who choose an extended cycle elect to "stack" 2-3 packs at a time; however, a continuous regimen can be used if desired with no hormone-free interval $[32,33]$. Women who prefer an extended cycle should be counseled regarding possible breakthrough bleeding. COCs require daily dosing. Providers should discuss compliance strategies with their patients, including finding the optimal time of day, paired activities (such as brushing teeth), phone alarms, reminder from family/friend, or use of birth control apps or text messaging services such as those found at www.bedsider.org.

The transdermal patch is a 1.75-in square that delivers $20 \mathrm{mcg}$ of ethinyl estradiol and $150 \mathrm{mcg}$ of norelgestromin daily. A patch is worn for 7 days for three consecutive weeks, followed by a patch-free week to allow for withdrawal bleeding. Patients may apply the patch on upper extremities, back, lower abdomen, or buttocks but not on the breasts. Patch can be worn with all normal daily activities, including bathing, swimming, and exercising. The patch delivers approximately $60 \%$ more estrogen than a 35-mcg pill over 21 days. Recent data suggests possible increased risk of VTE compared with COC, which should be considered when counseling patients, particularly those who prefer an extended cycle [34].

There has been conflicting data regarding diminished efficacy of the patch among overweight or obese females; recent data suggests this is not the case [35]. Current prescribing information still cautions use in women $>90 \mathrm{~kg}$ (198 lbs) [36].

Intravaginal ring

The vaginal ring (NuvaRing; Merck) is a flexible silicone ring measuring $5.4 \mathrm{~cm}$ ( 2 in) at the outer diameter with $4 \mathrm{~mm}$ thickness. It releases $15 \mathrm{mcg}$ of ethinyl estradiol and $120 \mathrm{mcg}$ of etonogestrel daily. The ring is inserted into the vagina for 3 weeks at a time, then removed for 7 days to allow for withdrawal bleeding. A new ring should then be inserted. 
The ring can be used with all normal daily activity, including bathing, swimming, exercise, and sexual intercourse. Patients should be counseled that if the ring is removed, it must be replaced within 3 hours to maintain optimal efficacy.

Extended cycle may be utilized with the vaginal ring.

\section{Progestin-only pills}

For women with contraindications to estrogen who prefer a pill, progestin-only pills (POP or "mini-pills") may be prescribed. POPs contain $0.35 \mathrm{mg}$ of norethindrone and are taken daily with no hormone-free days. Most women achieve decreased menstrual bleeding and $10 \%$ achieve complete amenorrhea. Breakthrough bleeding is the most common side effect.

POPs have the shortest half-life of any of the OCPs (approximately $21 \mathrm{~h}$ ) and require very accurate daily dosing. As a result, they are considered a less effective form of oral contraceptive in comparison to the combined pills and should not be used as first-line choice of contraceptive agents.

Several forms of emergency contraception (EC) are available currently in the USA, including single-dose levonorgestrel that is available over-the-counter without prescription or age limits. Other oral regimens include single-dose ulipristal (Ella; Watson) or high-dose combined COC (Yuzpe method). Each of these can be taken up to 5 days $(120 \mathrm{~h})$ after unprotected sexual intercourse, although they are most effective when taken as soon as possible. Placement of a copper IUD is considered the most effective form of emergency contraception, but requires access to a trained provider within 5 days of unprotected intercourse.

While access to emergency contraception has improved with over-the-counter availability of levonorgestrel EC, barriers still exist, including cost and accessibility. Advance prescription for EC can still be provided, which may increase access when needed and may also be a more cost-effective alternative to OTC. Discussion about EC should occur at each visit when providing anticipatory guidance strategies regarding safe sex practices.

EC delays ovulation. Counseling should include a warning that repeated unprotected sex shortly after administration of EC may still result in unintended pregnancy.

\section{Barrier contraception, other pregnancy prevention strategies}

As no form of hormonal contraception is considered $100 \%$ effective, barrier protection is encouraged with all sexual activity. Barrier methods such as male or female condoms also provide protection against sexually transmitted infections. OTC spermicides may offer additional contraceptive benefit. . While not considered an effective pregnancy prevention strategy, up to $60 \%$ of teenagers report the use of withdrawal method at least once [7]. See Table 2 for more detail. 


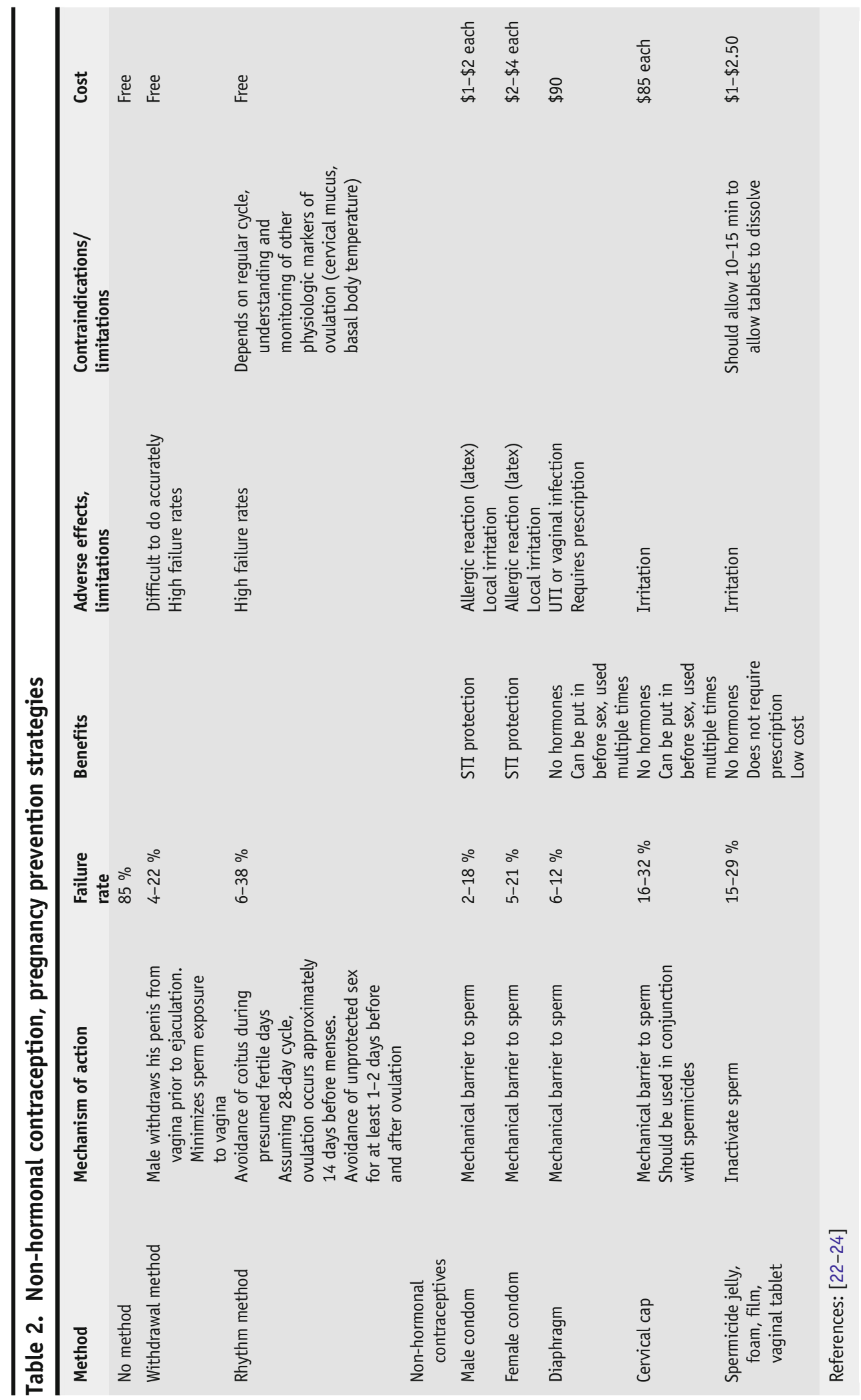




\section{Conclusions}

Sexual activity among teens is common. While most teens have used contraception at least once, the majority do not use effective contraception with all sexual activity. Clinicians who care for adolescents need to regularly screen for contraceptive use and counsel on available contraceptive methods, with an emphasis on the most effective LARC methods. An understanding of these methods, efficacy rates, and familiarity with prescribing practices and/or local resources for referral of ongoing contraceptive management is needed by all clinicians who serve adolescents.

\section{Compliance with Ethical Standards}

\section{Conflict of Interest}

Sarah Mermelstein and Katie Plax declare that they have no conflict of interest.

Human and Animal Rights and Informed Consent

This article does not contain any studies with human or animal subjects performed by any of the authors.

\section{References and Recommended Reading}

1. Hamilton BE, Martin JA, Osterman MJK, Curtin SC. Births: preliminary data for, 2014. National vital statistics reports; 64(6). Hyattsville: National Center for Health Statistics; 2015.

2. Santelli JS et al. Explaining recent declines in adolescent pregnancy in the United States: the contribution of abstinence and improved contraceptive use. Am J Public Health. 2007;97(1):150-6.

3. Statistics Canada. Table 102-4503 - Live births, by age of mother, Canada, provinces and territories, annual, CANSIM (database).

4. Kost, K., \& Henshaw, S. (2014). U.S. teenage pregnancies, births and abortions, 2010: National trends by age, race and ethnicity. Guttmacher Institute.

5. Abma M. Teenagers in the U.S.: sexual activity, contraceptive use, and childbearing, national survey of family growth 2006-2008. Vital Health Stat. 2010;23(30):147.

6. Perper K, Peterson K, Manlove J. Diploma attachment among teen mothers. Washington, DC: Child Trends; 2010. Retrieved March 2010.

7. Pinzon JL, Jones VF. Care of adolescent parents and their children. Pediatrics. 2012;130(6):e1743-56.

8. Ruedinger E, Cox JE. Adolescent childbearing: consequences and interventions. Curr Opin Pediatr. 2012;24(4):446-52.
9. Martinez GM, Abma JC. Sexual activity, contraceptive use, and childbearing of teenagers aged 15-19 in the United States. NCHS data brief, no 209. Hyattsville: National Center for Health Statistics; 2015.

10. Kann L, Kinchen S, Shanklin SL, Flint KH, Hawkins J, Harris WA, et al. Youth risk behavior surveillance-United States, 2013. MMWR Surveill Summ. 2014;63(4):1-168.

11. Guttmacher Institute State Center., available from: http://www.guttmacher.org/statecenter/adolescents. html.

12. Center for Adolescent Health and the Law. State minor consent laws: a summary. 3rd ed. Chapel Hill: Center for Adolescent Health and the Law; 2010.

13. Zabin LS, Stark HA, Emerson MR. Reasons for delay in contraceptive clinic utilization. Adolescent clinic and nonclinic populations compared. J Adolesc Health. 1991;12(3):225-32.

14. English A, Ford CA. The HIPAA privacy rule and adolescents: legal questions and clinical challenges. Perspect Sex Reprod Health. 2004;36(2):80-6.

15. Satterwhite CL et al. Sexually transmitted infections among U.S. women and men: prevalence and incidence estimates, 2008. Sex Transm Dis. 2013;40(3):187-93. 
16. WHO Medical eligibility criteria for contraceptive use. Fifth edition, 2015. http://apps.who.int/iris/bitstream/ 10665/181468/1/9789241549158_eng.pdf?ua=1

(Accessed on 23 Jan 2016).

17. Centers for Disease Control and Prevention, U.S. Medical eligibility criteria for contraceptive use, 2010 (US MEC). http://www.cdc.gov/mmwr/preview/ mmwrhtml/rr5904a1.htm?s_cid=rr5904a1_e (Accessed on 23 Jan 2016).

18. Birgisson N, Zhao Q, Secura G, Madden T, Peipert J. Preventing unintended pregnancy: the contraceptive CHOICE project in review. J Women's Health. 2015;24(5):349-53.

19. American College of Obstetricians and Gynecologists. Committee opinion no. 539: adolescents and longacting reversible contraception: implants and intrauterine devices. Obstet Gynecol. 2012;120:983-8.

20. Committee on Adolescence. Contraception for adolescents. Pediatrics. 2014;134:e1244.

21. Mestad R, Secura G, Allsworth JE, et al. Acceptance of long-acting reversible contraceptive methods by adolescent participants in the Contraceptive CHOICE Project. Contraception. 2011;84(5):493-8.

22. Mermelstein S, Tycast S, Plax K. Adolescent medicine. In: White A, editor. The Washington manual of pediatrics. Lippincott: Williams and Wilkins; 2016.

23. Gemzell-Danielsson K, Berger C, Lalitkumar PGL. Emergency contraception-mechanisms of action. Contraception. 2013;87:300-8.

24. The national campaign to prevent teen and unplanned pregnancy. (Available from:) http://bedsider.org.

25. Westoff C, Kerns J, Morroni C, Cushman LF, Tiezzi L, Murphy PA. Quick start: novel oral contraceptive initiation method. Contraception. 2002;66(3):141-5.

26. Nexplanon [package insert]. Whitehouse Station: Merck \& Co, Inc.; 2011.
27. Gemzell-Danielsson K, Inki P, Boubli L, et al. Bleeding pattern and safety of consecutive use of the levonorgestrel-releasing intrauterine system (LNGIUS) - a multicentre prospective study. Hum Reprod. 2010;25(2):354-9.

28. Skyla [package insert]. Wayne: Bayer HealthCare Pharmaceuticals; 2013.

29. Birgisson NE, Zhao Q, Secura GM, Madden T, Peipert JF. Positive testing for Neisseria gonorrhoeae and Chlamydia trachomatis and the risk of pelvic inflammatory disease in IUD users. J Womens Health (Larchmt). 2015;24:354-9.

30. Zieman M, Hatcher R, Allen A. Managing contraception. Tiger: Bridging the Gap Foundation; 2015.

31. American College of Obstetricians and Gynecologists. Depot medroxyprogesterone acetate and bone effects. Committee opinion no. 602. Obstet Gynecol. 2014;123:1398-402.

32. Anderson FD, Gibbons W, Portman D. Long-term safety of an extended-cycle oral contraceptive (Seasonale): a 2-year multicenter open label extension trial. Am J Obstet Gynecol. 2006;195(1):92-6.

33. Benson LS, Micks EA. Why stop now? Extended and continuous regimens of combined hormonal contraceptive methods. Obstet Gynecol Clin N Am. 2015;42(4):669-81.

34. Cole JA, Norman H, Doherty M, Walker AM. Venous thromboembolism, myocardial infarction and stroke among transdermal contraceptive system users. Obset Gynecol. 2007;109(2 pt 1):339-46.

35. McNicholas C, Zhao Q, Secura G, Allsworth JE, Madden T, Peipert JF. Obstet Gynecol. 2013;121(3):58592 .

36. Ortho-Evra [package insert]. Titusville: Janssen Pharmaceuticals; 2001. 